\title{
Immunoglobulins with proteolytic activity as a biomarker of impaired humoral immune system in schizophrenia
}

\author{
Evgeny Ermakov \\ ICBFM SB RAS, Novosibirsk, Russia \\ NSU, Novosibirsk, Russia \\ evgeny_ermakov@mail.ru
}

\author{
Valentina Buneva \\ ICBFM SB RAS, Novosibirsk, Russia \\ NSU, Novosibirsk, Russia \\ buneva@niboch.nsc.ru
}

\author{
Georgy Nevinsky \\ ICBFM SB RAS, Novosibirsk, Russia \\ NSU, Novosibirsk, Russia \\ nevinsky@niboch.nsc.ru
}

\begin{abstract}
Schizophrenia is known to be associated not only with imbalance in the neurotransmitter systems but also with immune system dysregulation. In this work, we found that pure polyclonal IgG preparations of patients with schizophrenia possess histone hydrolyzing proteolytic activity. The presence of proteolytic activity of IgGs is evidence of impaired humoral immune system in schizophrenia, which can be used as a biomarker for immunophenotyping of patients.
\end{abstract}

Keywords - immunoglobulins, IgG, proteolytic activity, histones, schizophrenia

\section{Motivation and aim}

There is a plethora of evidence that inflammation and dysregulation of the immune system is associated with schizophrenia [1]. One of the important signs of impaired humoral immunity is the generation of catalytic antibodies (Abs) that not only binding antigens but also hydrolyze these molecules [2]. Catalytic Abs were found among the total pool of immunoglobulins in autoimmune and viral diseases. Recently, we detected catalytic IgGs hydrolyzing DNA and RNA in serum of patients with schizophrenia [3, 4]. The formation of such Abs can be associated with immunization with nucleic acids and their complexes with nucleosome and histones. Autoantibodies to DNA were found in patients with schizophrenia [5]. Therefore, we suggested that such Abs can hydrolyze not only DNA and RNA, but also histones.

The aim of this work was to investigate the ability to hydrolyze various human histones by polyclonal IgGs from serum of patients with schizophrenia.

\section{Methods}

We recruited 25 patients (11 men and 14 women) with a verified diagnosis of paranoid schizophrenia and 20 healthy donors (9 men and 11 women) in the study. IgG preparations were obtained by Protein-G affinity chromatography of the serum proteins. The purity and homogeneity of obtained $\mathrm{IgG}$ preparations was verified using SDS-PAGE with silver staining, western blotting and MALDI mass-spectrometry. Catalytic activity of IgGs were revealed by the degree of hydrolysis of human histones as a substrate. IgGs were incubated with the substrate for 20 hours. The products of the hydrolysis were revealed by $20 \%$ SDS-PAGE and Coomassie G250 staining as in [6]. The gels were analyzed by scanning and quantified using Image Lab 6.0 software (Bio-Rad, USA). Statistical analysis was carried out using the Mann-Whitney U-test and Spearman's rank correlation in the Statistica 10 software (StatSoft, USA).

\section{Results}

Screening analysis showed that IgG preparations of patients with schizophrenia possessed histone-hydrolyzing activity and are able to hydrolyze various histones with different efficiencies. Using strict generally accepted criteria, we proved that the activity was due to IgGs, and was not a consequence of the impurities of various canonical proteases. The level of histone hydrolyzing activity of IgG varied over a wide range (from 0 to $100 \%$ ). The level of histones hydrolysis by IgGs of patients with schizophrenia was statistically significantly higher $(\mathrm{p}<0.02)$ than in healthy donors. Also, the level of histone hydrolysis by IgGs was significantly different $(p<0.05)$ in patients with positive and negative symptoms. According to the results of a retrospective analysis, a positive correlation $(\mathrm{R}>0.45)$ was found between the level of histone hydrolysis and the level of DNA-hydrolyzing activity of antibodies in schizophrenia.

Thus, we found new evidence of impaired humoral immune system in schizophrenia. We suggest that histonehydrolyzing antibodies may play a positive role in schizophrenia pathogenesis because histone removal from circulation minimizes the immune response. But the catalytic activity of IgG reflects the degree of derangement of immune system; therefore, it can be used for immunophenotyping and stratification of patients with schizophrenia. Identification of subgroups of patients with severe immunological disturbances will allow them to recommend additional anti-inflammatory therapy.

\section{ACKNOWLEDGMENT}

This work was supported by RFBR (20-015-00156), by Russian State funded budget project of ICBFM SB RAS (AAAA-A17-117020210023-1) and scholarship of the President of the Russian Federation (SP-2258.2019.4).

\section{REFERENCES}

[1] Miller B.J., Goldsmith D.R. (2017). Neuropsychopharmacology, 42(1), 299-317.

[2] Nevinsky G.A., Buneva, V.N. (2003). Catalytic antibodies in healthy humans and patients with autoimmune and viral diseases. Journal of cellular and molecular medicine, 7(3), 265-276.

[3] Ermakov E.A., et al. (2015). DNA-hydrolysing activity of IgG antibodies from the sera of patients with schizophrenia. Open biology, 5(9), 150064

[4] Ermakov E.A., et al. (2018). Hydrolysis by catalytic IgGs of microRNA specific for patients with schizophrenia. IUBMB life, 70(2), 153-164.

[5] Smirnova L.P., et al. (2016). Zhurnal nevrologii i psikhiatrii imeni SS Korsakova, 116(4), 47-51.

[6] Baranova S.V., et al. (2019). Antibodies from the Sera of Multiple Sclerosis Patients Efficiently Hydrolyze Five Histones. Biomolecules, 9(11), 741 . 